\title{
A Comparison of Methodical Approaches to Fingerprinting of the Volatile Fraction from Winter Savory (Satureja montana)
}

\author{
Józef Rzepa, ${ }^{1}$ Mieczysław Sajewicz, ${ }^{1}$ Tomasz Baj, ${ }^{2}$ Patrycja Gorczyca, ${ }^{1}$ Magdalena Włodarek, ${ }^{1}$ \\ Kazimierz Głowniak, ${ }^{2}$ Monika Waksmundzka-Hajnos, ${ }^{3}$ and Teresa Kowalska ${ }^{1}$ \\ ${ }^{1}$ Institute of Chemistry, University of Silesia, 9 Szkolna Street, 40-006 Katowice, Poland \\ ${ }^{2}$ Department of Pharmacognosy with Medicinal Plant Unit, Faculty of Pharmacy, Medical University of Lublin, \\ 1 Chodźki Street, 20-093 Lublin, Poland \\ ${ }^{3}$ Department of Inorganic Chemistry, Faculty of Pharmacy, Medical University of Lublin, Collegium Pharmaceuticum, \\ 4a Chodźki Street, 20-093 Lublin, Poland \\ Correspondence should be addressed to Teresa Kowalska, teresa.kowalska@us.edu.pl
}

Received 14 August 2011; Accepted 3 October 2011

Academic Editor: Yvan Vander Heyden

Copyright (C) 2012 Józef Rzepa et al. This is an open access article distributed under the Creative Commons Attribution License, which permits unrestricted use, distribution, and reproduction in any medium, provided the original work is properly cited.

It was the aim of this study to compare the efficiency of the different essential oil extraction methods upon the two winter savory (Satureja montana) samples of different origin. The compared techniques were the headspace gas chromatography with mass spectrometric detection (HS-GC/MS) run at the two different headspace temperatures (i.e., at 80 and $100^{\circ} \mathrm{C}$ ) and the three different steam distillation techniques preceding the GC/MS analysis. HS-GC/MS is considered as the technique of the first choice, and the compared steam distillation techniques are recommended, respectively, by Polish Pharmacopoeia, European Pharmacopoeia, and the Polish Patent. Adequate conclusions were drawn as to the advantage of HS-GC/MS (not having the pharmacopoeial recommendation) over the different steam distillation techniques and the drawbacks of each individual analytical procedure were discussed.

\section{Introduction}

The genus Satureja L. contains over 30 species. Winter savory (Satureja montana) is a perennial plant belonging to the family Lamiaceae, growing mainly in the regions of South Europe. It is a semievergreen subshrub growing to about $50 \mathrm{~cm}$ tall with the oval-lanceolate leaves and white flowers. Satureja montana contains numerous subspecies, and there is much variability in morphologic characteristics of the species Satureja montana L. [1]. It is similar in use and flavor to the annual summer savory (Satureja hortensis) and it is cultivated as a culinary herb having spicy flavor. Both summer and winter savory have a long history of use in traditional medicine as tonics, carminatives, astringents, and expectorants, and for the treatment of intestinal problems such as diarrhea and nausea. However, the scientific literature primarily documents Satureja hortensis (and not Satureja montana L.) as a folk remedy in treating various ailments such as cramps, muscle pains, nausea, indigestion, diarrhea, and infectious diseases [1-3].
Winter savory contains ca. $1.6 \%$ volatile oil, whereas summer savory only ca. $1.0 \%$. Some authors document the dominant components of the volatile oil as caryophyllene and geraniol, or as carvacrol. The relative composition of the volatile oil varies with the location of cultivation, the species, and the strain [3-6]. The essential oil of the Satureja sp. has a broad spectrum of antimicrobial activity [7-10]. Satureja montana L. also has a potent anti-HIV-1 activity [11].

With this study on fingerprinting of the volatile fraction contained in Satureja montana, we continue our earlier commenced methodical approach to fingerprinting of the volatile fractions derived from the other medicinal and culinary plants belonging to the same family Lamiaceae. In our earlier studies (e.g., [12-15]), we have focused our attention on the selected representatives of the Salvia genus, the largest one in the Lamiaceae family. In spite of great popularity of many plants belonging to the Lamiaceae family both in traditional European medicine and the Mediterranean cuisine, an insufficient attention has been paid so far to fingerprinting 
of this particular botanical material. Fingerprints, that is, chromatographic profiles derived from the plant extracts, can provide a sufficient basis for differentiation of plants, their chemotaxonomic comparison, identification within a given family, and so forth. Moreover, they can be used for rapid screening of plant material to prevent the forgery in the bulk trading on the medicinal and culinary herbs. Due to the variation in the consistency of plant material both in the fresh and the dried form, the efficiency of the different extraction methods can also differ and hence, the best performing extraction technique has to be experimentally confirmed for each individual genus.

The main goal of this study was to make a methodical comparison of the performance of the basic fingerprinting strategies applied to the volatile fraction contained in the dried samples of Satureja montana. To this effect, we compared the headspace-GC/MS fingerprints of the volatile fraction (HS-GC/MS) and with the fingerprints originating from the three different steam distillation techniques preceding the GC/MS analysis (Figure 2). HS-GC/MS is generally considered as the technique of the first choice, and the compared steam distillation techniques are recommended, respectively, by Polish Pharmacopoeia [16], European Pharmacopoeia [17], and the Polish Patent [18]. Partial identification of the volatile fraction components was also performed.

\section{Experimental}

2.1. Herbal Material and Reagents. Two different samples of winter savory (Satureja montana) were investigated in this study. Lot 1 was harvested in Pharmacognosy Garden of the Medical University, Lublin, Poland. The plant material comprised all parts of the plant (i.e., roots and the aerial parts) and it was dried for $40 \mathrm{~h}$ in an oven with a forced air flow at 35 to $40^{\circ} \mathrm{C}$. Then the obtained dry material was stored in the deep-freeze compartment of refrigerator until the commencement of the analysis. Finally, plant material from lot 1 was weighed and ground with a mechanical blender. Lot 2 originated from the farm market in Belgrade (Serbia) as the dried herbs and it was not pretreated in our laboratory in any way, except for identical grinding as with lot 1 .

The following solvents were used in this study: methanol (HPLC purity grade, P.O.CH, Gliwice, Poland) and water (double distilled and deionized with use of the Elix Advantage model Millipore system, Molsheim, France).

2.2. Headspace Gas Chromatography-Mass Spectrometry (HSGC/MS) of the Volatile Fraction. The headspace gas chromatography-mass spectrometry (HS-GC/MS) analyses were carried out with use of a TRACE 2000 model GC with an MS TRACE model mass detector (ThermoQuest, Waltham, MA, USA), equipped with a CTC Analytics model autosampler (Combi PAL, Basel, Switzerland), used in the headspace mode. Temperatures and time of the headspace desorption were, respectively, 80 and $100^{\circ} \mathrm{C}$, and $15 \mathrm{~min}$. The $0.5 \mathrm{~mL}$ volume of the headspace phase was introduced on to the DB-5 capillary column $(30 \mathrm{~m} \leftrightarrow 0.25 \mathrm{~mm}$ i.d., $0.25-\mu \mathrm{m}$ film thickness; Agilent Technologies, Palo Alto, CA, USA).
Helium ( $p=100 \mathrm{kPa}$ ) was used as carrier gas. Gradient analysis was run using the following temperature program: $40^{\circ} \mathrm{C}(1 \mathrm{~min}) ; 40-180^{\circ} \mathrm{C}\left(12^{\circ} \mathrm{C} / \mathrm{min}\right)$; and $180^{\circ} \mathrm{C}(20 \mathrm{~min})$. The temperature of the injector was kept constant at $180^{\circ} \mathrm{C}$. Mass spectrometer was fitted with an EI source operated at $70 \mathrm{eV}$. Identification of individual compounds was based on a comparison of the obtained mass spectra of the individual chromatographic peaks with those valid for the standards and available from the National Institute of Standards and Technology software library (Gaithersburg, MD, USA). A comparison was also carried out of the retention times valid for individual peaks from the Satureja montana samples with those of the known essential oils components. To this effect, we used pine oil, peppermint oil, eucalyptus oil, juniper oil, thyme oil, and lavender oil as the sets of the volatile fraction standards (Apotheca Pacis, Rybnik, Poland). The identified compounds originating from lots 1 and 2 are listed in Table 1.

2.3. Steam Distillation Modes. In this study, we compared the performance of the three steam distillation modes, as given below:

(a) mode 1, applied to the lot 1 and lot 2 samples (50 g) with use of the Deryng apparatus recommended by Polish Pharmacopoeia VI [16];

(b) mode 2, applied to the lot 1 sample (50 g) with use of the Clevenger apparatus recommended by European Pharmacopoeia [17];

(c) mode 3(a), applied to the lot 1 sample (20 g) with use of the Clevenger apparatus (like in Mode 2);

(d) mode 3(b), applied to the lot 1 sample $(20 \mathrm{~g})$ with use of the Clevenger apparatus, yet with the herbal/aqueous mixture additionally ultrasonicated in order to enhance maceration of herbal material, as recommended by the Polish Patent [18].

In the experiments valid for modes 1 and 2, the dried plant material $(50 \mathrm{~g})$ was placed in the round-bottomed flask, $400 \mathrm{~mL}$ water was added, and the steam distillation was performed for $3 \mathrm{~h}$. In the experiments valid for modes 3(a) and 3(b), aimed to compare the efficiency of the Clevenger apparatus without and with ultrasonication, the $20 \mathrm{~g}$ amounts of the plant material with $400 \mathrm{~mL}$ water was placed in the round-bottomed flask, $400 \mathrm{~mL}$ water was added, and the steam distillation was performed for $3 \mathrm{~h}$.

From the distillates obtained in each individual experiment, the $5 \%(v / v)$ solutions in methanol were prepared and the $1-\mu \mathrm{L}$ aliquots of the respective solutions were analyzed by means of GC/MS. The applied chromatographic conditions were the same as those given in Section 2.2.

\section{Results and Discussion}

This study was focused on a comparison of the efficiency of the methods used for derivation and fingerprinting of the volatile fraction from winter savory (Satureja montana). To this effect, we compared the results originating from the method of the first choice (which is HS-GC/MS) 


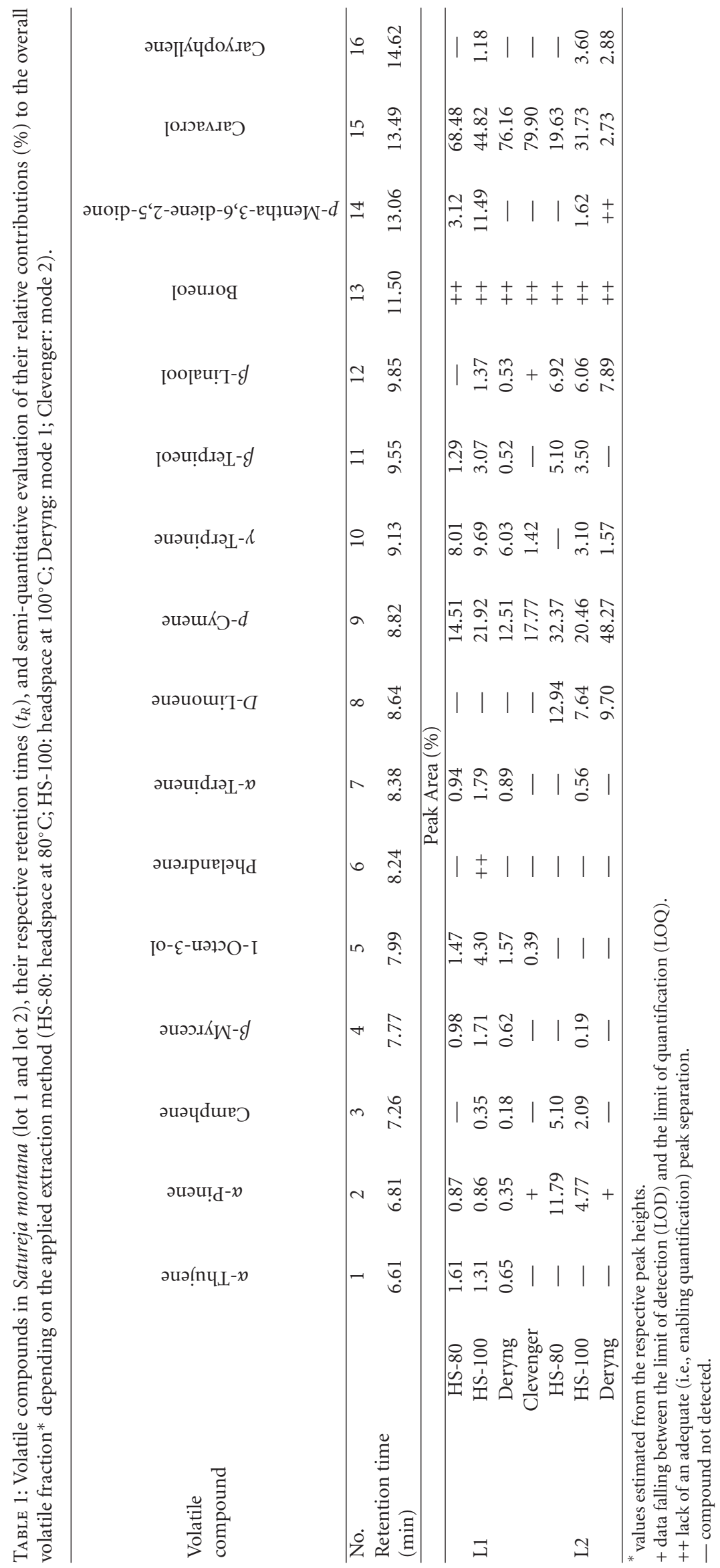


with those of the steam distillation of the volatile fraction (recommended by many national and international pharmacopoeias), followed by the GC/MS analysis. Our results originate from the HS-GC/MS experiment with the two different headspace temperatures and from the three different steam distillation modes. All experiments presented in this study were performed twice, upon the two different batches of plant material from lot 1 and lot 2. Due to a limited amount of the available plant material and hence, due to a limited number of repetitions, the quantitative results presented in this study were taken as the mean values, but not statistically evaluated. Although these results bear a semi-quantitative importance only, the estimated relative percent error of quantification of the individual volatile compounds (based on the two repetitions) never surpassed the $\pm 3 \%$ level. In the forthcoming paragraphs, the obtained results are going to be presented and discussed.

In Table 1, all volatile compounds identified and semiquantitatively assessed in the analyzed Satureja montana samples (lots 1 and 2) are listed, depending on the applied extraction mode. The most frequently appearing volatile compounds were $\alpha$-pinene, $p$-cymene, $\gamma$-terpinene, terpineol, $\beta$-linalool, borneol, and carvacrol, as shown in Table 2 (together with the respective structural formulas and mass spectra). These results remain in good agreement with those known from the literature (e.g., $[19,20])$.

From the general phytochemical knowledge and also from the obtained results it is evident that the origin of the plant material (and also its preprocessing like, e.g., the drying technique) plays a meaningful role in chemical composition of the volatile fraction. Thus, in lot 1 (originating from Pharmacognosy Garden of the Medical University in Lublin, Poland), $\alpha$-thujene and 1-octen-3-ol were identified, which were absent from lot 2 (originating from the farm market in Belgrade, Serbia). To the contrary, D-limonene was identified exclusively in lot 2 and it was not detected in lot 1 . These differences are evidently due to the different origin (in terms of the different cultivation regions, the climatic and strain differences, the different drying techniques, etc.) of the two examined specimens.

A comparison of analytical results dealing with the headspace extraction of the volatile fraction carried out at 80 and $100^{\circ} \mathrm{C}$ allowed drawing very practical conclusions. The chromatograms addressing this particular issue are given in Figure 1 (chromatograms (a) versus (b) valid for lot 1 and chromatograms (e) versus (f) valid for lot 2). A comparison of the aforementioned chromatograms showed that at the higher headspace temperature $\left(100^{\circ} \mathrm{C}\right)$ a higher number of the volatile compounds were isolated than at the lower one. Thus applying the headspace temperature of $100^{\circ} \mathrm{C}$, in lot 1 camphene, phellandrene, $\beta$-linalool, and caryophyllene were identified, which were not extracted from the same plant sample at $80^{\circ} \mathrm{C}$. When applying the headspace temperature of $100^{\circ} \mathrm{C}$ to lot $2, \beta$-myrcene, $\alpha$ terpinene, $\gamma$-terpinene, $p$-mentha-3,6-diene-2,5-dione, and caryophyllene were isolated, which were absent from the volatile fraction extracted from the same plant sample at the lower headspace temperature.
A consecutive comparison was made of the analytical results valid for the headspace extraction of the volatile fraction carried out at $100^{\circ} \mathrm{C}$ with that obtained for the steam distillation in the Deryng apparatus (mode 1). The conclusions can be drawn from a comparison of the chromatograms shown in Figures 1(b) and 1(c) (lot 1, HS100 versus mode 1), and of those shown in Figures 1(f) and $1(\mathrm{~g})$ (lot 2, HS-100 versus mode 1$)$. This comparison allows a conclusion that the headspace extraction carried out at $100^{\circ} \mathrm{C}$ is more effective particularly with the more volatile compounds than the steam distillation in the Deryng apparatus. From a comparison of the data contained in Table 1 and valid for the two investigated S. montana samples (lot 1 and lot 2), it is evident that the percent contributions of the more volatile compounds (i.e., those with the relatively low retention times, $t_{R}$ ) are considerably higher, when the headspace extraction is carried out at $100^{\circ} \mathrm{C}$ than with the steam distillation in mode 1 .

Then the two steam distillation techniques with use of the Deryng and the Clevenger apparatus (modes 1 and 2) are compared for lot 1 (Figure 1, chromatograms (c) versus (d)). Essential oil derived with use of the Deryng apparatus (mode 1, Figure 1(c)) proved richer in terms of the isolated and identified chemical species than that derived with use of the Clevenger apparatus (mode 2, Figure 1(d)). Namely, in the essential oil extracted with use of the Clevenger apparatus, only four compounds were identified (i.e., 1octen-3-ol, $p$-cymene, $\gamma$-terpinene, and carvacrol), while in the essential oil isolated with use of the Deryng apparatus, six additional compounds (i.e., $\alpha$-thujene, camphene, $\beta$ myrcene, $\alpha$-terpinene, $\beta$-terpineol, and $p$-mentha-3,6-diene2,5 -dione) were found. This experimental outcome can be due to the construction differences of these two steam distillation apparatuses, and specifically to the less efficient reflux condensation system in the Clevenger apparatus, allowing an easier loss of the most volatile compounds than the Deryng apparatus.

The last comparison refers to the contents of the volatile compounds in the samples belonging to lot 1 and originating from the steam distillation in the Clevenger apparatus without and with ultrasonication (derivation modes 3(a) and 3(b), resp.). It was shown that the steam distillation combined with ultrasonication resulted in a lower concentration yield with one essential oil component ( $p$ cymene) and in an absence of the two components (1octen-3-ol and $\gamma$-terpinene; see Table 3 ), as compared with the distillation without ultrasonication. The only compound which showed slightly higher extraction yield in the case of steam distillation supported with ultrasonication was carvacrol, one of the least volatile compounds on the list (98.54\% contribution to the overall volatile fraction obtained in mode $3(\mathrm{~b})$ versus $92.97 \%$ obtained in mode $3(\mathrm{a})$ ). This result is probably due to the too long ultrasonication period (equal to $180 \mathrm{~min}$ ), although from the literature it comes out that the steam distillation yields remain practically constant even with the two or more hours lasting ultrasonication (although the shortest recommended ultrasonication period is 20 minutes; [21]). Thus with Satureja montana, maceration of plant material with use of ultrasonication cannot 
TABLE 2: Seven volatile compounds most frequently appearing in Satureja montana with their respective structural formulas and mass spectra.

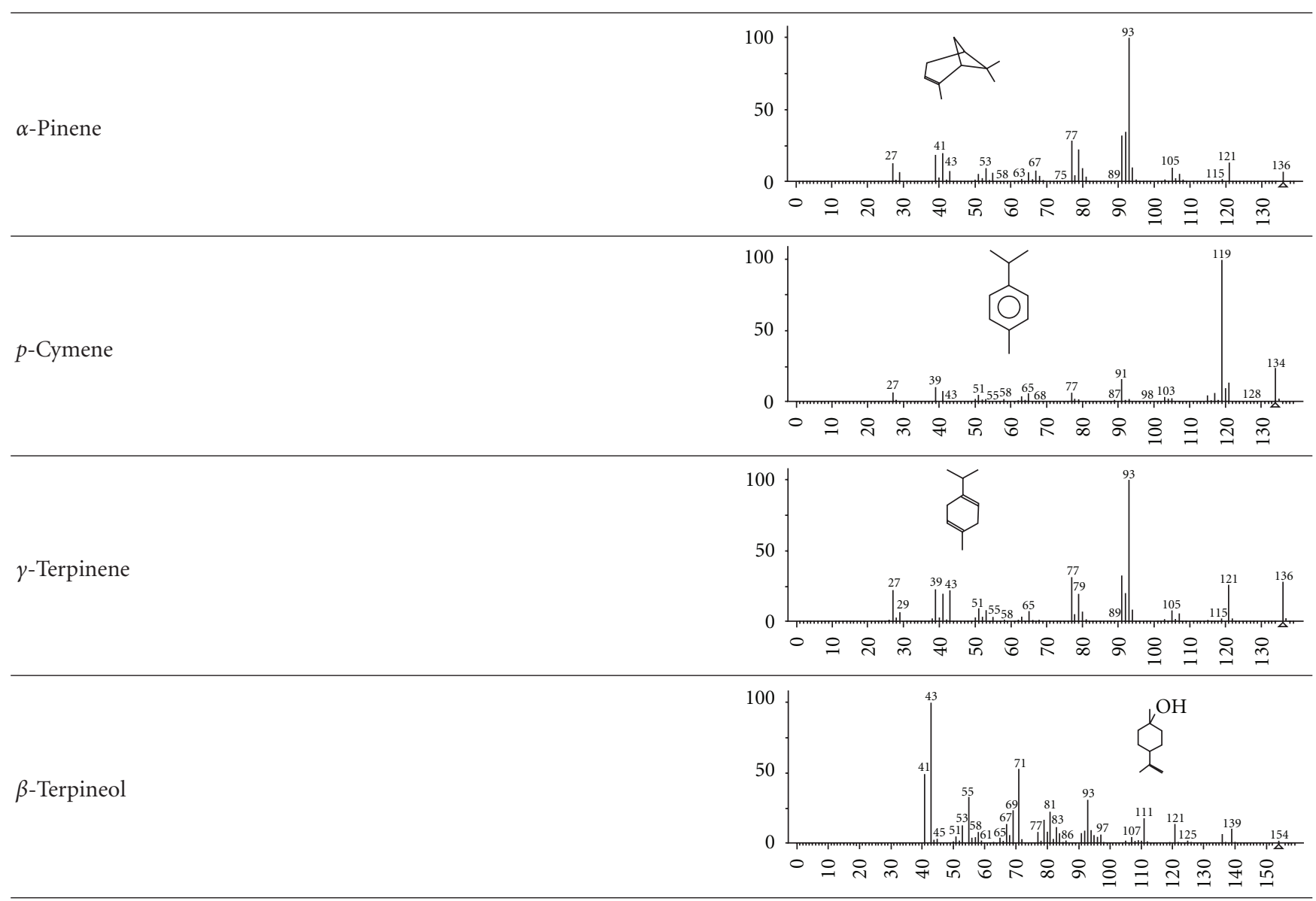

$\beta$-Linalool

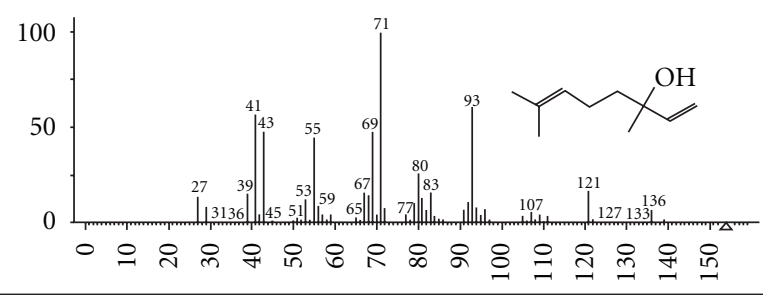

Borneol

Carvacrol

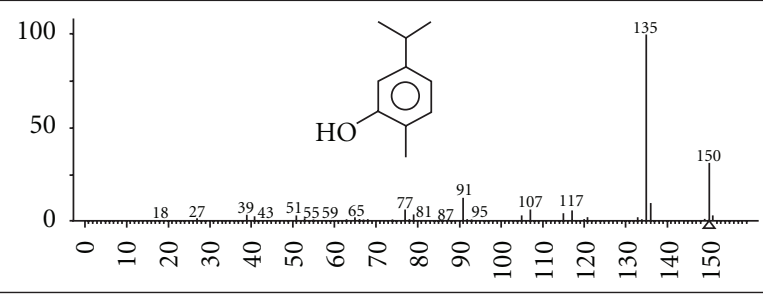




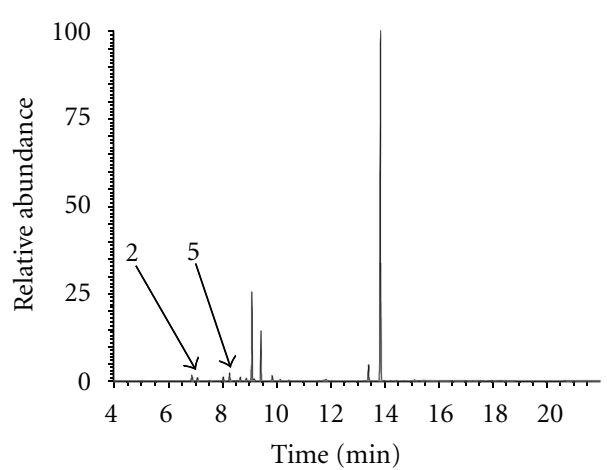

(a)

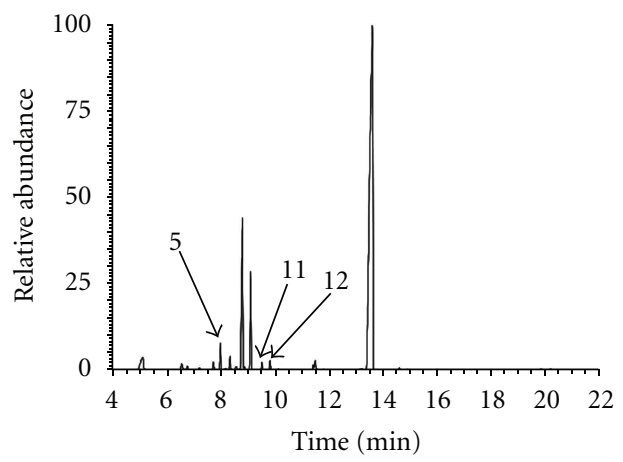

(c)

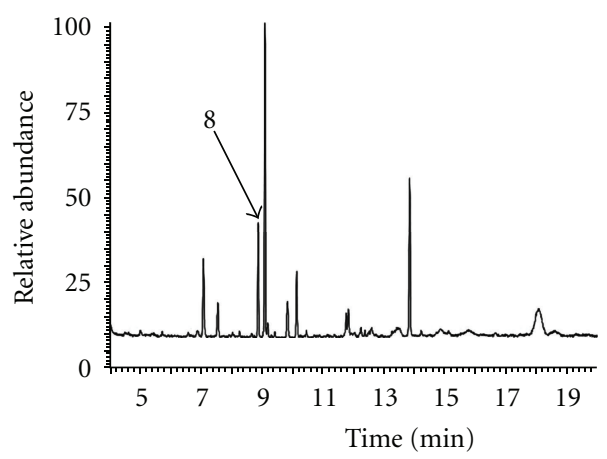

(e)

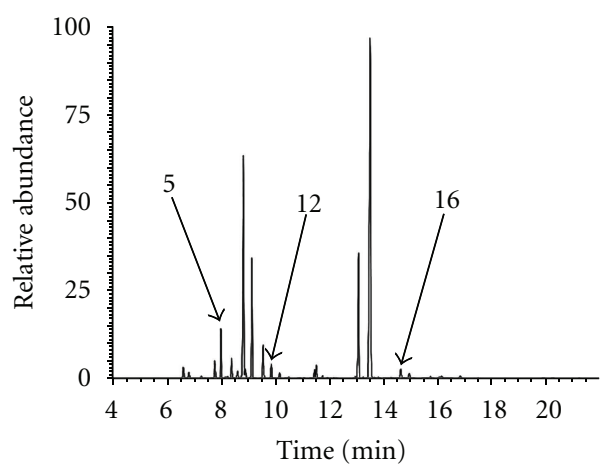

(b)

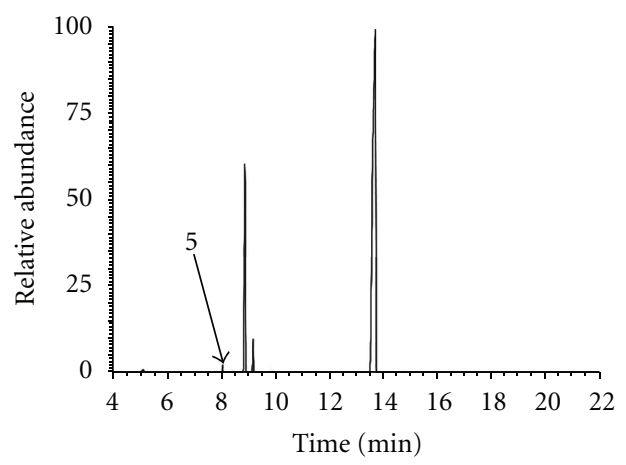

(d)

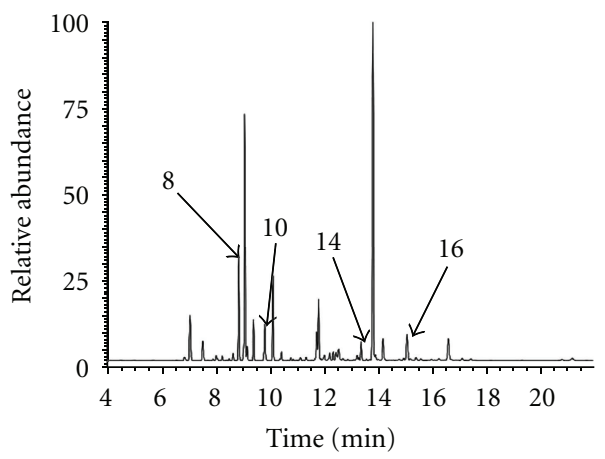

(f)

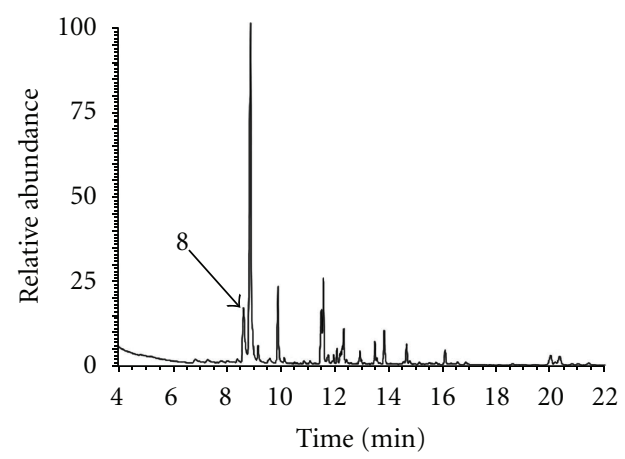

(g)

Figure 1: The GC/MS fingerprints of the volatile Satureja montana fraction for the following samples: (a): Lot 1, HS-80; (b): Lot 1, HS-100; (c): Lot 1, Deryng; (d): Lot 1, Clevenger; (e): Lot 2, HS-80; (f): Lot 2, HS-100; (g): Lot 2, Deryng. Extraction modes and peak numbers remain in conformity with those given in Tables 1 and 2. 


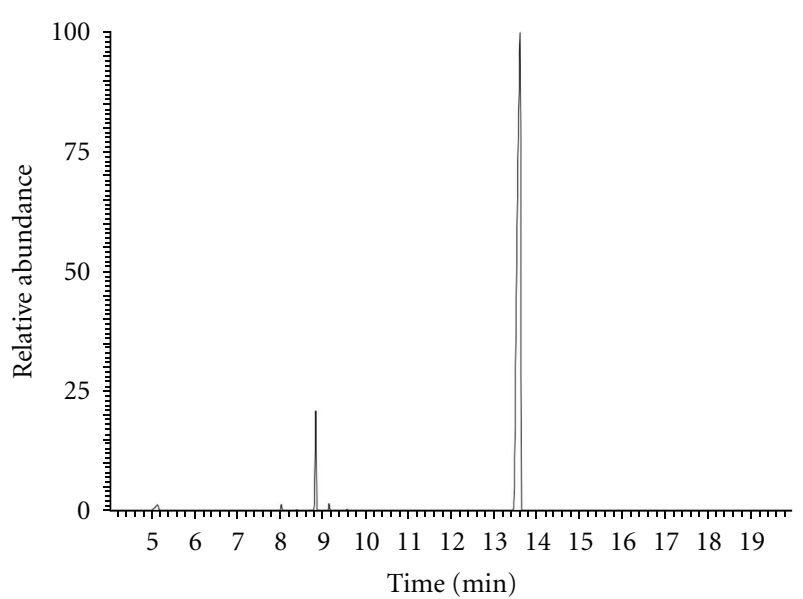

(a)

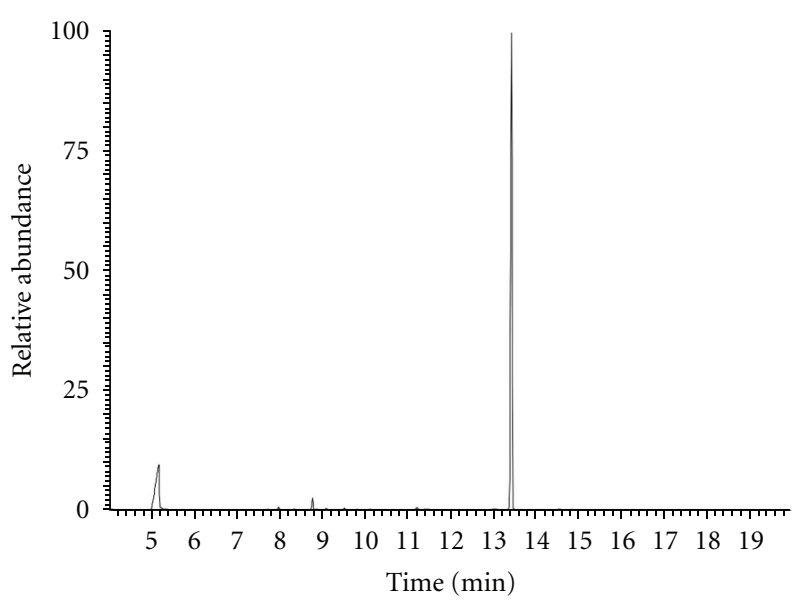

(b)

FIGURE 2: The GC/MS fingerprints of the volatile Satureja montana fraction (lot 1) vapor distilled with use of the Clevenger apparatus. (a) without ultrasonication; (mode 3(a) and 3(b)) with ultrasonication (mode 3(b)).

Table 3: Volatile compounds in Satureja montana (lot 1), their respective retention times $\left(t_{R}\right)$, and semiquantitative evaluation of their relative contributions (\%) to the overall volatile fraction* depending on the applied extraction method (mode 3(a) and mode 3(b)).

\begin{tabular}{lcccc}
\hline Volatile compound & 1-Octen-3-ol & p-Cymene & $\gamma$-Terpinene & Carvacrol \\
\hline No. ${ }^{*}$ & 5 & 9 & 10 & 15 \\
Retention time (min) & 8.01 & Peak Area (\%) & 9.12 & 13.56 \\
\hline & & 0.31 & 0.40 & 92.97 \\
\hline Mode 3(a) & 0.32 & 0.19 & + & 98.54 \\
\hline
\end{tabular}

* values estimated from the respective peak heights.

** numbering of the volatile compounds in conformity with Table 1.

+ data falling between the limit of detection (LOD) and the limit of quantification (LOQ).

be recommended, because the loss of the more volatile essential oil components is in this case higher than without ultrasonication.

\section{Conclusions}

The methodical aspects of the analysis of the volatile fraction contained in winter savory (Satureja montana) were discussed in this study and they can be summarized in the following way.

(i) It was demonstrated that the headspace desorption of the volatile fraction run at $100^{\circ} \mathrm{C}$ outperforms the steam distillation modes with use of the Deryng apparatus, the Clevenger apparatus, and the Clevenger apparatus in combination with ultrasonication of the hydrodistilled material.

(ii) This result is most probably due to an uncontrolled loss of the most volatile components both from the Deryng apparatus and the Clevenger apparatus (as an evident partial "leaking" of the two reflux condensation systems).

(iii) Furthermore, it was shown that this uncontrolled loss (both in qualitative and quantitative terms) is somewhat higher with the Clevenger than the Deryng apparatus.

(iv) Additionally, it was shown that the ultrasonicationsupported maceration of plant material belonging to the S. montana species in the Clevenger apparatus results in an even greater overall loss of the volatile fraction components than without using this supplementary technique.

(v) In order to make phytochemical analysis of the volatile fractions contained in medicinal plants more up to the date and in the first instance considerably more accurate, it seems recommendable (or even urgent) to replace pharmacopoeial hydrodistillation techniques with the headspace-GC/MS technique (as a standard separation and quantification tool in modern analytical chemistry laboratories).

\section{References}

[1] P. Schauenberg and F. Paris, Guide to Medicinal Plants, Keats Publishing, New Canaan, Conn, USA, 1990.

[2] C. Uslu, R. M. Karasen, F. Sahin, S. Taysi, and F. Akcay, "Effects of aqueous extracts of Satureja hortensis L. on rhinosinusitis treatment in rabbit," Journal of Ethnopharmacology, vol. 88, no. 2-3, pp. 225-228, 2003. 
[3] J. A. Duke, CRC Handbook of Medicinal Herbs, CRC Press, Boca Raton, Fla, USA, 1985.

[4] V. Slavkovska, R. Jancic, S. Bojovic, S. Milosavljevic, and D. Djokovic, "Variability of essential oils of Satureja montana L. and Satureja kitaibelii Wierzb. ex Heuff. from the central part of the Balkan peninsula," Phytochemistry, vol. 57, no. 1, pp. 71-76, 2001.

[5] M. De Vincenzi, A. Stammati, A. De Vincenzi, and M. Silano, "Constituents of aromatic plants: carvacrol," Fitoterapia, vol. 75, no. 7-8, pp. 801-804, 2004.

[6] A. Radonic and M. Milos, "Chemical composition and in vitro evaluation of antioxidant effect of free volatile compounds from Satureja montana L," Free Radical Research, vol. 37, no. 6, pp. 673-679, 2003.

[7] M. Güllüce, M. Sökmen, D. Daferera et al., "In vitro antibacterial, antifungal, and antioxidant activities of the essential oil and methanol extracts of herbal parts and callus cultures of Satureja hortensis L," Journal of Agricultural and Food Chemistry, vol. 51, no. 14, pp. 3958-3965, 2003.

[8] F. Şahin, I. Karaman, M. Güllüce et al., "Evaluation of antimicrobial activities of Satureja hortensis L," Journal of Ethnopharmacology, vol. 87, no. 1, pp. 61-65, 2003.

[9] M. Ciani, L. Menghini, F. Mariani, R. Pagiotti, A. Menghini, and F. Fatichenti, "Antimicrobial properties of essential oil of Satureja montana L. on pathogenic and spoilage yeasts," Biotechnology Letters, vol. 22, no. 12, pp. 1007-1010, 2000.

[10] K. Oberg, L. Rolling, and C. Oberg, "Selection of essential oil components to inhibit Candida without affecting normal microbiota," The Journal of the Utah Academy of Sciences, Arts, and Letters, vol. 82, pp. 60-72, 2005.

[11] K. Yamasaki, M. Nakano, T. Kawahata et al., "Anti-HIV-1 activity of herbs in Labiatae," Biological and Pharmaceutical Bulletin, vol. 21, no. 8, pp. 829-833, 1998.

[12] J. Rzepa, Ł. Wojtal, D. Staszek et al., "Fingerprint of selected salvia species by HS-GC-MS analysis of their volatile fraction," Journal of Chromatographic Science, vol. 47, no. 7, pp. 575-580, 2009.

[13] M. Sajewicz, J. Rzepa, M. Hajnos et al., "GC-MS study of the performance of different techniques for isolating the volatile fraction from sage (Salvia L.) species, and comparison of seasonal differences in the composition of this fraction," Acta Chromatographica, vol. 21, no. 3, pp. 453-471, 2009.

[14] M. Sajewicz, L. Wojtal, D. Staszek, M. Hajnos, M. Waksmundzka-Hajnos, and T. Kowalska, "Low temperature planar chromatography-densitometry and gas chromatography of essential oils from different sage (Salvia) species," Journal of Liquid Chromatography and Related Technologies, vol. 33, no. 7-8, pp. 936-947, 2010.

[15] M. Sajewicz, Ł. Wojtal, M. Natić, D. Staszek, M. Waksmundzka-Hajnos, and T. Kowalska, "TLC-MS versus TLC-LC-MS fingerprints of herbal extracts. Part I. essential oils," Journal of Liquid Chromatography and Related Technologies, vol. 34, no. 10-11, pp. 848-863, 2011.

[16] Polish Pharmacopoeia VI, Polish Pharmaceutical Society, Warsaw, Poland, 2002.

[17] European Pharmacopoeia, Vol. 3, pp.68, Maisonneuve SA, Sainte Ruffine, France, 1975.

[18] Polish Patent No 208058, 2008.

[19] G. S. Ćetković, A. I. Mandić, J. M. Čanadanović-Brunet, S. M. Djilas, and V. T. Tumbas, "HPLC screening of phenolic compounds in winter savory (Satureja montana L.) extracts," Journal of Liquid Chromatography and Related Technologies, vol. 30, no. 2, pp. 293-306, 2007.
[20] J. Masteli and I. Jerkovi, "Gas chromatography-mass spectrometry analysis of free and glycoconjugated aroma compounds of seasonally collected Satureja montana L," Food Chemistry, vol. 80, no. 1, pp. 135-140, 2003.

[21] R. Kowalski and J. Wawrzykowski, "Effect of ultrasoundassisted maceration on the quality of oil from the leaves of thyme Thymus vulgaris L," Flavour and Fragrance Journal, vol. 24, no. 2, pp. 69-74, 2009. 


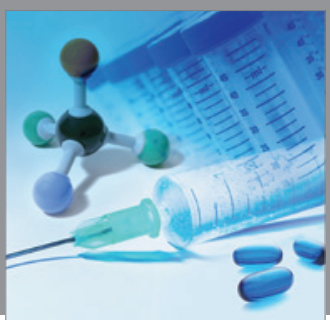

International Journal of

Medicinal Chemistry

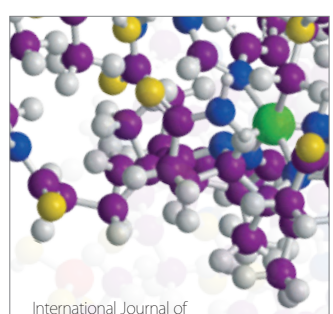

Carbohydrate Chemistry

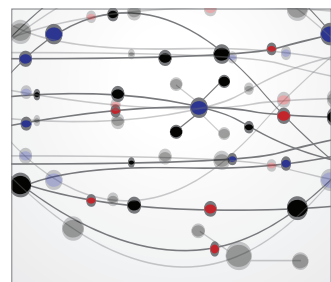

The Scientific World Journal
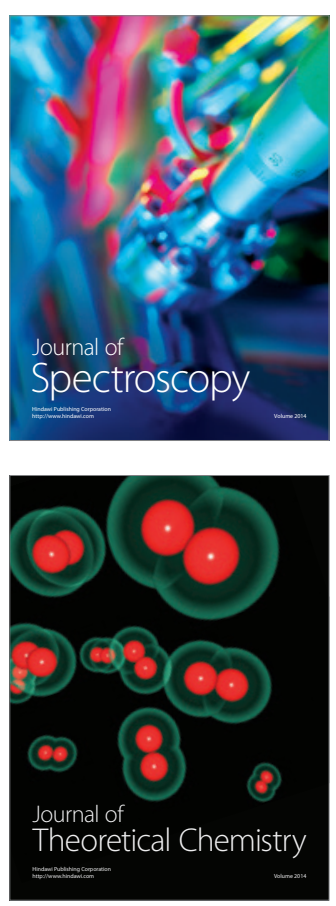
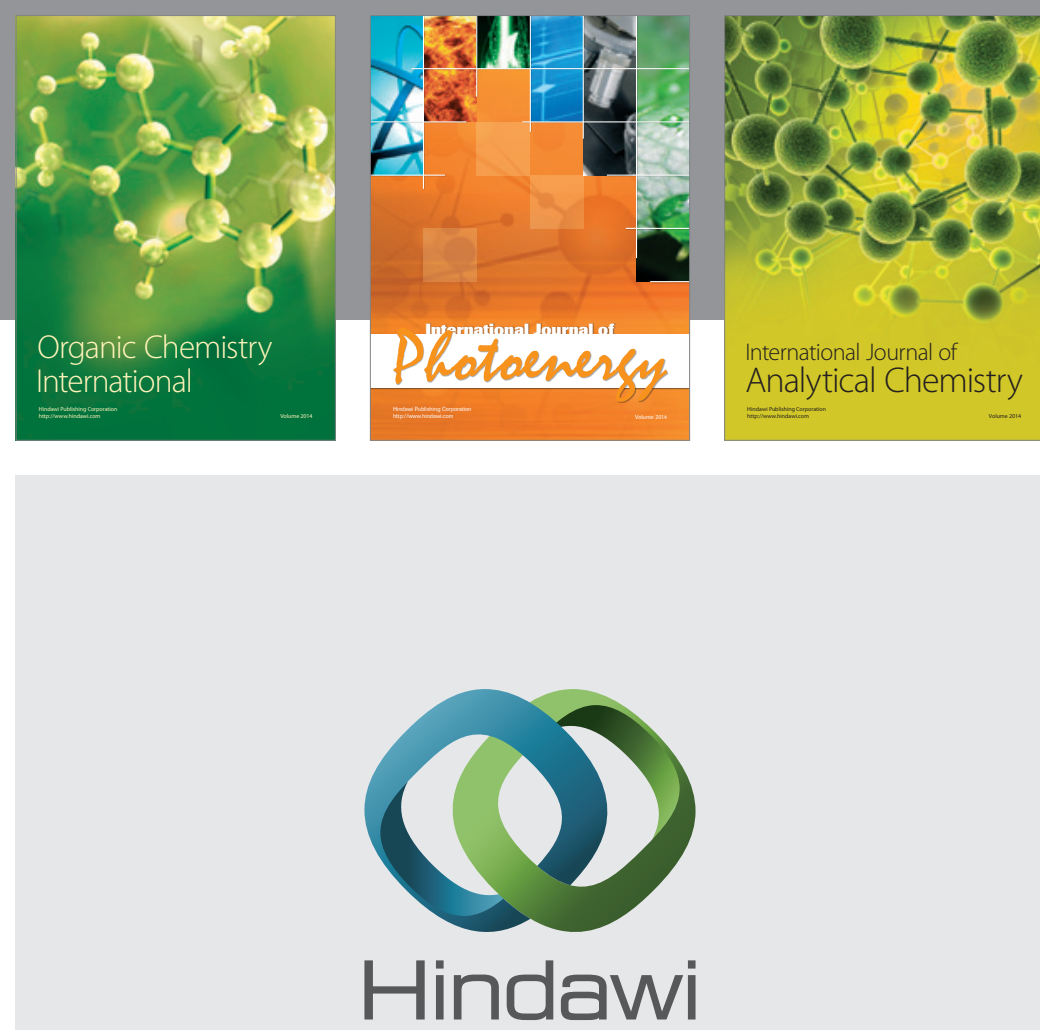

Submit your manuscripts at

http://www.hindawi.com
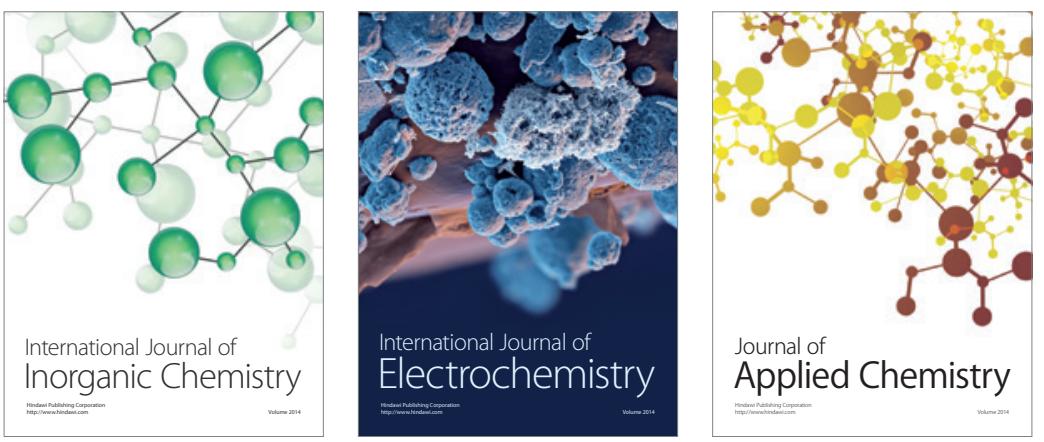

Journal of

Applied Chemistry
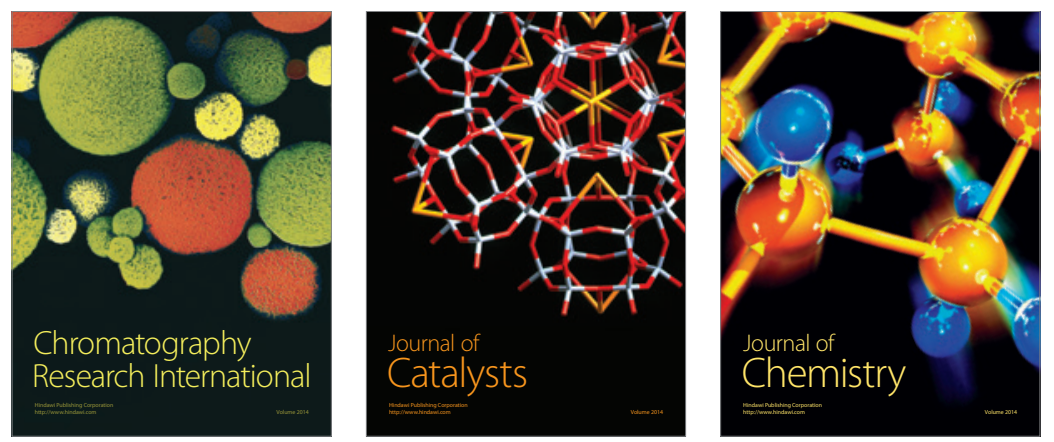
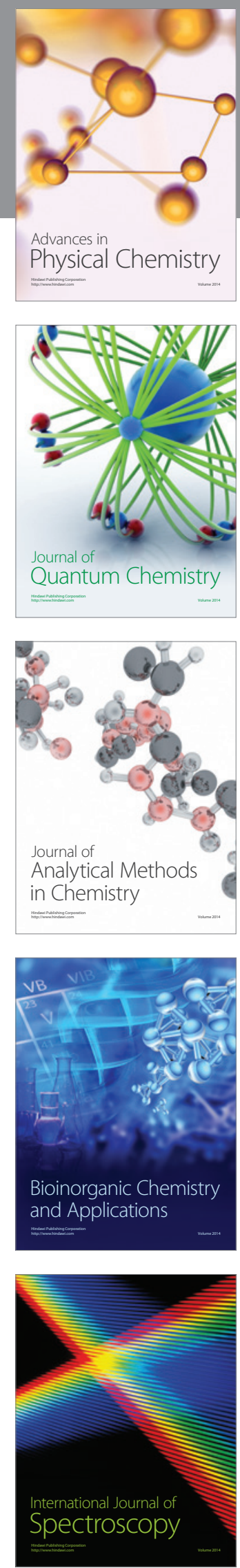